\title{
Ripensare Socrate: note su alcuni studi recenti
}

Livio Rossetti, Le dialogue socratique, Les Belles Lettres, Paris 2011 e Livio Rossetti, Alessandro Stavru (a cura di), Socratica 2008, Atti Convegno Napoli 2008, Le Rane, Levante, Bari 2011.

\section{FULVIA DE LUISE / Trento /}

\section{Un approccio innovativo alla questione socratica}

L'uscita quasi contemporanea di due volumi a nome di Livio Rossetti (la raccolta Le dialogue socratique per Les Belles Lettres e Socratica 2008 per l'editrice Levante) è un evento che merita una riflessione più ampia di una semplice recensione. Nel primo caso si tratta di un volume che seleziona e raccoglie in versione francese otto saggi, relativamente recenti, tra i moltissimi che lo studioso ha dedicato al lavoro about Socrates. Nel secondo caso, si tratta degli Atti di un convegno (curati da Rossetti insieme ad Alessandro Stavru) svoltosi a Napoli nel 2008, parte di una serie cominciata nel 2005 a Senigallia, che intende riproporsi a ritmo triennale come appuntamento fisso per gli studi socratici (intervallando le scadenze dei Symposia platonici promossi dalla International Plato Society).

L'interesse scientifico di questa doppia pubblicazione sta nel fatto che essa offre una sorta di bilancio in corso d'opera di una stagione di studi davvero sorprendente: una "rinascenza" socratica che ha radici nell'intenso lavoro compiuto, tra gli anni Cinquanta e gli Ottanta, sulle fonti socratiche diverse da Platone'; che passa per gli studi anali-

${ }^{1}$ Si tratta di una stagione di studi culminante nella pubblicazione delle Socraticorum reliquiae prima (19831985) e delle Socratis et Socraticorum reliquiae poi (1990), da parte di Gabriele Giannantoni, caratterizzata da 
tici degli anni Novanta, particolarmente significativi per la rivalutazione di Senofonte (Morrison, Dorion, Narcy) e di Antistene (Rankin e Brancacci); che infine, negli ultimi dieci anni, sembra aver preso la forma di una svolta metodologica, in grado di ridisegnare il quadro degli studi di settore, riproponendo in termini radicalmente nuovi la vexata quaestio dell'identità intellettuale di Socrate e determinando una svolta anche nel campo degli studi platonici. È un rethinking Socrates, vorrei dire (citando uno dei temi chiave di queste raccolte di studi), che non solo ripopola di presenze differenziate l'ambiente della recezione antica più vicina alla vita e alla morte del maestro (accettandone l'irriducibile gioco di specchi), ma cambia significativamente anche la recezione attuale dei dialoghi platonici, consegnati da una lunga tradizione a uno splendido (e fuorviante) isolamento.

Per segnalare gli elementi di novità che ridefiniscono l'asse interpretativo di alcune questioni cruciali, possiamo cominciare dal Rossetti promotore dell'iniziativa dei Socratica. Attingo per questo al suo intervento di apertura alle giornate di Senigallia nel 2005, I socratici della prima generazione, che addita all'interesse generale l'area di dibattito che si raccoglie intorno a Socrate e ne trasmette in forma plurima la memoria. Ormai acquisite (anche, in buona parte, per merito del suo lavoro) l'entità e la rilevanza del fenomeno letterario dei logoi sokratikoi, Rossetti ha buon gioco nel farne il punto di partenza per una revisione di metodo, invertendo, in prima battuta, il pregiudizio sorto dalla pluralità differenziata delle immagini socratiche: non si tratta di dichiarare irraggiungibile il Socrate storico e di accettare la sua riduzione a leggenda (rassegnandosi a condividere lo scetticismo di Olof Gigon sulla possibilità di trascendere la finzione dei dialoghi); si tratta invece di prendere sul serio l'aspetto mimetico che necessariamente si accompagna alle rappresentazioni di Socrate, come personaggio noto e dibattuto davanti al pubblico antico, ben riconoscibile attraverso e oltre l'attribuzione di particolari coloriture e contenuti al suo insegnamento. Poiché «l'imitazione suppone l'avvenuta individuazione di tratti rappresentativi del personaggio», è importante considerare questo aspetto «una risorsa per risalire al modello imitato»; e il quadro rappresentativo fornito dalla letteratura socratica antica «è assai stabile nel caratterizzare Socrate come un personaggio che si impegna volentieri a costruire situazioni dialogiche tali da generare negli interlocutori perplessità e imbarazzo, in modo da lasciar balenare idee o istanze suscettibili di tradursi in esortazione per l'interlocutore» (LR Socratica 2005, p. 47).

Naturalmente, l'operazione che consente di cogliere tratti di stabilità tra le rappresentazioni dei socratici è già stata più volte tentata (soprattutto a proposito di Platone e Senofonte); e l'attenzione al modo di dialogare di Socrate, invece che ai contenuti delle sue conversazioni, ha sollecitato interpretazioni penetranti, come quelle di Giannantoni e di Vlastos. Ma Rossetti ha il merito di costruire connessioni inedite tra aspetti noti, traendone significative implicazioni metodologiche: sottolineando, per esempio, che

un'attenzione alle fonti dell'eredità socratica, che ha prodotto un completo rinnovamento nell'approccio ai cosiddetti "socratici minori". Importanti studiosi (tra cui lo stesso Giannantoni, Olof Gigon, Fernanda Decleva Caizzi, Leo Strauss, Andreas Patzer, Klaus Döring, Luciano Montoneri) hanno contribuito ad aprire nuove vie di ricerca in questa direzione. 
i tratti di riconoscibilità del personaggio Socrate (principalmente, il suo spiazzante modus operandi nei confronti degli interlocutori) siano allo stesso tempo i tratti identificativi di un gruppo intellettuale assai agguerrito nel manipolare e superare forme comunicative ben collaudate sul finire del V secolo (trattati e testi paradossali, modelli teatrali di dialogo e di azione), col risultato di riversare nella forma di un nuovo genere (i logoi sokratikoi) la vocazione a "fare cose con le parole".

Ciò induce a considerare l'insieme di questa straordinaria produzione di unità dialogiche come un modo di agire comune nello spazio della parola pubblica, ma anche e prima di tutto come un modo di intervenire nell'area del dibattito interno tra i socratici, dove le differenze si implicano reciprocamente come parti di un dialogo conflittuale. Somiglianze e differenze nei contenuti delle rappresentazioni risultano così ugualmente illuminanti per comprendere la posta in gioco: l'eredità condivisa e contesa, in parte enigmatica, di ciò che era possibile dire nel nome di Socrate, come primo referente di ogni specifica (e postuma) elaborazione.

La fecondità di un tale innovativo approccio alla questione socratica emerge a mio parere con chiarezza nella progressione tematica che si è espressa nello spazio dei convegni socratici, da Senigallia 2005 a Napoli 2008. Dall'invito a prendere sul serio i "socratici minori", per ricomporre l'unità di ispirazione che li coinvolge tutti (posto in exergo agli Atti di Senigallia 2005 e di Napoli 2008, attraverso una citazione di Romeyer Dherbey 2001), alla chiara messa a fuoco della rilevanza di ciò che accade entro la cerchia di quella prima generazione di socratici per il futuro della filosofia: perché essa si affermi, innanzitutto, come pratica intellettuale distinta da ogni forma già codificata di sapere. Nel saggio che apre la raccolta degli atti di Socratica 2008 (I Socratici 'primi filosofi' e Socrate 'primo filosofo'), Rossetti compie in effetti un passo importante riesaminando, analiticamente e comparativamente, tutte le occorrenze dei termini "filosofia" e "filosofo" nel V secolo, per collocare precisamente a carico della prima generazione dei socratici, la scelta di dare un significato intensivo a quei termini, identificando con essi Socrate e la sua pratica discorsiva. Ciò lega indissolubilmente l'invenzione della filosofia al dialogo reale con cui Socrate si impose come affascinante innovatore nel panorama culturale di Atene nell'ultimo trentennio del V secolo; e lega i socratici (not Plato alone!) all'ideazione di quella forma di scrittura che avrebbe perpetuato l'immagine di Socrate come emblema irriducibile della filosofia in azione, quali che fossero i contenuti veicolati e trasmessi in suo nome. Lascio ai lettori del saggio il piacere di cogliere nelle sottili argomentazioni di Rossetti le implicazioni di questa tesi per una rilettura critica degli schemi storiografici trasmessi dalla tradizione (con l'indicazione aristotelica in testa, che pone gli autori di scritti Peri physeos come primi filosofi), per una rinnovata comprensione della frattura tra filosofi e sofisti (postulata in diversi modi dai socratici a difesa della memoria del maestro e di se stessi), per delimitare lo spazio temporale delle accuse ai "filosof", cui accennano Senofonte e Platone. Ciò che mi sembra importante sottolineare è che lo spostamento del focus sui socratici ha il valore di un piano di ricerca, e che le scoperte in questo ambito, di interesse non solo storiografico, rendono inevitabile una consistente revisione degli schemi interpretativi usati dalla tradizione filosofica per pensare se stessa. 


\section{Socratica 2008: uno sguardo sul panorama degli studi socratici recenti}

Nell'Introduction a Socratica 2008, i curatori tracciano un ampio quadro del rinnovamento degli studi che segue la pubblicazione delle Socratis et Socraticorum Reliquiae di Giannantoni, sottolineando che l'effetto si manifesta con evidenza alla distanza di un decennio, da considerarsi come «a period of rumination» (p. 12). Non può stupire un certo ritardo nell'utilizzo pieno della miniera di fonti vecchie e nuove, messe a disposizione dalla raccolta: resta un'impresa non priva di difficoltà la ricostruzione del valore reale di un'esperienza come quella dei socratici, che ha subito alle origini un processo di quasi completa rimozione. Come già notava Vegetti nel suo intervento a Socratica 2005, la solitudine di Socrate è un mito difficile da rimuovere, legato com'è alla brillante resa drammatica della rappresentazione platonica, che esalta insieme la grandezza e la marginalità di Socrate rispetto al contesto cittadino, oscurando del tutto il ruolo dei socratici. Ed è soprattutto a causa dello straordinario successo dei dialoghi platonici nell'antichità, che si spiega l'eclissi dei socratici e la sostanziale dispersione dei loro scritti, inattingibili per noi (con la sola eccezione di Senofonte) nella loro interezza.

«When Plato started writing dialogs, presumably shortly after 399 B.C.», notano i curatori, «his elder companions as Antisthenes or Aeschines enjoyed far higher status than he did. Fifty years later, in 350, the situation had completely changed, as the superiority of Plato - of his writings as well as of his school - was an undisputed fact». Ma quale rilievo ha oggi riaprire la discussione su quel giudizio selettivo della storia, che ha dato alla tradizione il suo orientamento, sommergendo e quasi cancellando la memoria dei socratici, in quanto filosofi? Il primo motivo di interesse sta forse proprio nel comprendere le ragioni per cui la versione platonica di Socrate, che orientò la tradizione ad assumere un determinato modello di filosofia in suo nome, ebbe una tale prevalenza: «a closer look at the theoretical issues of the 'minor' Socratics is necessary for a true and full acknowledgement of Plato's superiority» (p. 16).

La suggestione si fa più ricca e filosoficamente interessante se proviamo a immaginare i possibili sviluppi di una rinnovata attenzione al contesto in cui Platone ha scritto i suoi dialoghi. Alcuni di tali sviluppi possono considerarsi già praticati e ben rappresentati nel vivo delle ricerche in corso, di cui Socratica 2008, come diremo meglio in dettaglio, offre una gamma di importanti esempi:

1. una migliore focalizzazione di Socrate, come fonte di una novità intellettuale recepita e riverberata da tutte le sue rappresentazioni;

2. la messa in luce, o la riscoperta, di prospettive di ricerca divergenti dalla comune matrice socratica, oscurate dalla prevalenza del modello platonico (filone già ricco di risultati importanti nei casi di Senofonte e di Antistene);

3. una seria considerazione dell'interesse suscitato nei contemporanei dalla figura di Socrate, da vivo e da morto, che ne fa un personaggio di alto rilievo politico, poi un'eredità capace di dividere, ma anche un tramite di alleanze, di ricostruzioni, di revisioni della memoria storica, nel contesto della potenza ateniese in declino. 
Un ulteriore motivo, di carattere metodologico, rende a mio parere desiderabile la rottura dell'isolamento del teatro platonico dei dialoghi: la possibilità di una comprensione intertestuale della scrittura platonica, come stesura d'autore in dialogo con altre scritture, decifrabile per somiglianze e differenze, in luoghi topici e dettagli di stile. Questa prospettiva potrebbe andare a costituire il campo di applicazione più promettente per un ritorno a Platone attraverso Socrate e i socratici. In questo senso interpreto la novità della scelta tematica indicata per Socratica 2012 (terzo simposio socratico, previsto a Trento per il prossimo febbraio, di cui è partito a luglio il call for papers): «An International Conference on Socrates, the Socratics, and every thing that is around Plato».

Più in generale, mantenendo vivo l'interesse per l'area di dibattito che si raccoglie intorno a Socrate, e costituisce poi il milieu in cui si muove l'elaborazione platonica, i nuovi studi socratici permettono di muoversi verso una comprensione più concreta e dialettica delle grandi questioni etiche che ricevettero una formulazione in quel contesto: volontarietà-involontarietà del male; significato dei piaceri (o dei criteri di utilità) nell'ambito di una vita che si vuole felice; la "virtù" come ambiguo termine di riferimento, tra oggettivazione tecnica e processi di (ri)soggettivazione del valore; carattere e "destino" degli individui; forme di vita e forme di relazionalità pubblica; appartenenza, atopia e esperienze di straniamento nella costituzione del soggetto morale. L'attenzione al contesto presenta l'ulteriore vantaggio di valorizzare le implicazioni storico-politiche di tali questioni (consegnate altrimenti a una monumentale perennità), restituendo freschezza e attualità alle soluzioni teoriche disputate e ai percorsi impiegati per raggiungere soluzioni accettabili. Ciò significa, per esempio, cogliere nel teatro platonico dei dialoghi l'ambizione di allargare alla città il dibattito interno all'area socratica, aprendo un confronto retrospettivo con le voci più forti, autorevoli o deprecabili, di un passato recente e ancora carico di ombre sul presente politico della polis; e assumere definitivamente il carattere non fittizio di tali dialoghi, che proprio nella ricostruzione di quel milieu culturale (e degli interrogativi che in esso si andavano aprendo) pongono lo sfondo necessario a comprendere il gesto atopico di Socrate, la pratica irriverente e spiazzante che tutti i socratici hanno visto nel maestro, ma forse il solo Platone ha trasformato nel paradigma del filosofo e del fare filosofia.

Torniamo più analiticamente al panorama di studi presentato in Socratica 2008.

Come la precedente edizione e altri recenti convegni di argomento socratico, la raccolta mostra, nell'ampiezza e nella varietà dei percorsi delineati dai relatori, l'autenticità della "ripartenza" negli studi degli ultimi dieci anni, l'urgenza del re-thinking Socrates come fenomeno di portata internazionale, che trae, dalla nuova consapevolezza delle dimensioni eclatanti della produzione dei logoi, la necessità di riaprire il discorso sulla pratica intellettuale che riceve il nome di "filosofia": nome impegnativo e autorevole per la tradizione futura, ma, all'epoca, neologismo vivo e sfuggente, fonte di ispirazione e di contese, di definizioni e scritture ancora in corso. Spicca, nel panorama delineato con cura analitica e straordinaria ricchezza di riferimenti nell'Introduction agli atti del convegno, l'emergere di nuovi nuclei di condensazione dell'interesse degli studiosi: la reale portata della ricerca naturalistica di Socrate e dei suoi esiti teleologici (tema che 
induce a rileggere e incrociare le divergenti testimonianze di Aristofane, di Senofonte e di Platone); la varietà dei legami attribuiti a Socrate (per esempio, con Euripide o con Archelao) come indizi di una personalità culturale complessa, irriducibile a un ritratto univoco (considerazione che induce a valorizzare la peculiarità delle tracce socratiche più deboli, ordinariamente schiacciate dalle testimonianze più forti, e a mantenere attiva la pluralità dei resoconti sugli stessi argomenti); la riabilitazione di Senofonte, come socratico dotato di un'autonoma personalità filosofica, ben riconosciuta dagli antichi come alternativa a Platone, e titolare di una quota significativa, benché sottovalutata, del socratismo moderno (una linea di ricerca ben consolidata in area francofona, che vede ora in crescita il numero degli studiosi di diverse aree linguistiche interessati a scandagliare la complessità degli apporti senofontei su argomenti di rilievo filosofico per la tradizione occidentale); infine, come si è più volte accennato, la rilettura di Platone attraverso l'insieme delle testimonianze socratiche (prospettiva che si incrocia con l'esigenza di una revisione degli schemi adottati per distinguere l'autore dal suo personaggio, per maneggiare l'insieme dei dialoghi come un sistema unitario o in sviluppo, per contestualizzare temi topici come l'ironia o la religiosità di Socrate). Aggiungerei a quest'ultima prospettiva l'interesse a rileggere la biografia intellettuale di Socrate attraverso il resoconto distribuito da Platone nei dialoghi: certo una narrazione non neutrale e un palinsesto del suo programma filosofico, ma anche una ricostruzione in qualche modo realistica, perché esposta al confronto con la memoria degli altri socratici.

\section{Gli interventi in dettaglio}

Gli interventi raccolti si distribuiscono in quattro aree calde di dibattito (I - The first generation; II - Plato; III - Xenophon; IV - Nachleben), per concludersi con il ricordo In Memoriam di Mario Montuori.

La prima sezione, dedicata a ciò che accade tra i socratici e alla sua rilevanza per l'elaborazione multifaceted del paradigma del filosofo, comprende i saggi di Livio Rossetti, Noburu Notomi, Aldo Brancacci e Domingo Placido.

\ Livio Rossetti discute, come si è già detto, le occorrenze dei termini philosophia e philosophos tra V e IV secolo, per prospettare una tesi di rilevante interesse sulla formazione di una determinata identità intellettuale nel gruppo dei socratici.

$\checkmark$ Noburu Notomi discute i termini dell'opposizione canonica tra sofisti e filosofi nella letteratura socratica, a partire dal confronto tra le diverse strategie difensive adottate da Platone e Senofonte nei confronti del valore del maestro, per concludere sottolineando che l'enfasi a dividere Socrate dai sofisti, ineliminabile dal quadro della tradizione filosofica, appartiene specificamente al solo Platone ed è rivolta a criticare, insieme ai sofisti, i socratici come Antistene.

« Aldo Brancacci fa parlare il catalogo delle opere antisteniche trasmesso da Diogene Laerzio, ricostruendo il nesso tra moralità e sapere che caratterizza la ricerca del socratico e fornendo, attraverso una ricchissima disamina delle fonti, una 
risposta definitiva sul tipo di proposta etica che Antistene derivava dalla sua più lunga e antica frequentazione di Socrate: un concetto di episteme strettamente legato alla dimensione dialogico-dialettica, del tutto alieno dal riferimento a idee di tipo platonico, capace di conferire un fondamento unitario a logica ed etica sulla base della distinzione tra oikeion e allotrion.

\ Domingo Placido utilizza le diverse fonti relative a Eschine per formulare ipotesi sul retroterra economico di alcune apparenti contraddizioni del socratismo, collegando, in particolare, le differenze di atteggiamento nei confronti dell'insegnamento professionale a pagamento di tipo sofistico alle condizioni materiali di esistenza dei discepoli, che vanno dalla ricchezza di un aristocratico come Platone all'indigenza di Eschine, più vicino forse alle condizioni di vita del maestro.

La seconda sezione, dedicata alla rilettura di aspetti della rappresentazione platonica, comprende i saggi di Louis-André Dorion, Walter Omar Kohan, Lidia Palumbo, Gabriele Cornelli e André Leonardo Chevitarese.

$\checkmark$ Louis-André Dorion argomenta sulla differenza rilevabile tra il Socrate di Senofonte e quello di Platone in tema di autarkeia, rintracciando i motivi che rendono indesiderabile per Platone quella che in Senofonte appare virtù eminente del filosofo: uno stato di autosufficienza, incompatibile con il ruolo positivo che Platone assegna allo stato di bisogno, come base per l'intreccio di relazioni economiche, di amicizia e d'amore, e come condizione per lo sviluppo filosofico del desiderio di sapere.

« Walter Omar Kohan si muove all'interno dei dialoghi platonici, sottolineando alcune caratteristiche paradossali del personaggio Socrate, enigmaticamente sospeso tra la professione di ignoranza e l'impegno a curarsi degli altri come un autentico maestro di virtù, forse legato in modo essenziale al paradosso di un'educazione filosofica intesa come impegno a "decolonizzare” il proprio pensiero.

\ Lidia Palumbo procede alla revisione del celebre luogo platonico dell'Alcibiade I, in cui Socrate invita Alcibiade alla «cura di sé», utilizzando la metafora dell'occhio che si specchia nell'occhio per vedersi, per sostenere che il suo significato non è un invito alla relazione con l'altro, ma ad instaurare un dialogo dell'anima con se stessa, cioè un'autentica esperienza di «riflessione».

« Gabriele Cornelli e André Leonardo Chevitarese ripropongono l'analisi dei motivi che portarono alla morte di Socrate, riunendo sinergicamente il punto di vista e gli strumenti di indagine della storia e quelli della filosofia, per pervenire a un confronto realistico tra le ragioni politiche della condanna e quelle della canonizzazione filosofica.

La terza sezione, dedicata al Socrate di Senofonte come altra versione di ciò che significa filosofare, comprende i saggi di Donald Morrison e di Alessandro Stavru.

« Donald Morrison sviluppa l'analisi dei diversi tipi di connessione, rilevabili nei Memorabili di Senofonte, tra sophia e virtù, e sulla difficoltà a ricondurli a un modello unitario, come la fiducia nell'infallibilità del sapere per la conquista del 
bene, che richiede numerose sotto-ipotesi per raccordarsi con la relatività del proprio bene, con la limitatezza del sapere umano, con la pluralità delle forme di virtù, che in ogni caso includono un fondamento nel sapere.

\ Alessandro Stavru analizza un discusso passo del III libro dei Memorabili, in cui il Socrate senofonteo costringe gli artisti Parrasio e Clitone a misurarsi sullo statuto dell'immagine artistica, sul significato del rappresentare e del far apparire, dimostrando, con una ben documentata ricostruzione, che il dialogo evidenzia una precisa competenza in materia di canoni e procedimenti delle arti figurative del suo tempo da parte di Senofonte, unita alla capacità di tradurre e rielaborare i contenuti del linguaggio tecnico in chiave speculativa.

La quarta sezione, dedicata alla rinascita di temi socratici nell'antichità, comprende i saggi di Michael Erler, Graziano Ranocchia, Michel Narcy,

々 Michael Erler dedica il saggio alla ripresa in ambito epicureo del tema socratico della parrhesia, che viene sviluppato nella pluralità delle sue accezioni, attraverso un articolato confronto con i luoghi platonici che ne sanciscono la rilevanza, ma ne limitano l'uso a determinati contesti e condizioni pedagogiche.

〈 Graziano Ranocchia analizza il ritratto di Socrate come «eiron», citato da Filodemo nel De vitiis come parte dell'opera De superbia di un imprecisato Aristone, discutendo l'origine di un testo pesantemente denigratorio e caricaturale nei confronti dei tratti ironici del personaggio Socrate, in un contesto culturale in cui questi venivano identificati con la tradizione platonico-scettica, per concludere identificando l'autore nello stoico Aristone di Chio, in quanto esponente della scuola che si richiamava a un Socrate non ironico attraverso la divergente filiazione senofontea.

\ Michel Narcy discute l'accostamento tra Socrate e Euripide, proposto da Diogene Laerzio sulla base di un frammento comico, indagando le intenzioni rappresentative del dossografo nel dare spazio a una voce originariamente denigratoria nei confronti di Euripide, per rovesciarne il significato a favore di Socrate.

\section{Il contributo di Rossetti allo studio del dialogare socratico: un'immagine inquietante}

Passiamo ora dagli effetti a largo raggio dell'iniziativa socratica di Livio Rossetti al suo contributo personale di studioso, ben rappresentato dalla raccolta Le dialogue socratique, curata dallo psicoanalista francese François Roustang, a sua volta affascinato dalla figura di Socrate come tipo umano e autore di un libro dall'eloquente titolo Le secret de Socrate pour changer la vie (2009).

Il volume, che appare evidentemente un omaggio alla posizione di prestigio di cui il lavoro di Rossetti gode a livello internazionale, raccoglie in realtà solo un piccolo campione dei suoi scritti: 8 saggi (di cui 3 tradotti dall'italiano e 5 già apparsi in francese), tratti dalla produzione relativamente recente (tra il 1995 e il 2010) di uno studioso il cui 
primo scritto in materia socratica risale al 1971 (Recenti sviluppi della questione socratica, in Proteus 6, pp. 177-182). A fare da cornice all'insieme, troviamo, in apertura, il saggio «Le dialogue socratique in statu nascenti», che inquadra nelle sue dimensioni epocali il fenomeno della letteratura socratica; in chiusura, il saggio presentato a Socratica 2008, dedicato, come si è detto, al nesso tra esperienza socratica e nascita della filosofia.

Presentando nell'Avant-propos il filo rosso della raccolta, il curatore segnala il contributo fornito da Rossetti alla riformulazione, e in un certo senso alla soluzione, della questione socratica, rappresentata dall'interrogativo 'chi è il vero Socrate nella pluralità delle sue rappresentazioni?'. Usando un'accettabile semplificazione, si può dire con Roustang che, essendo Socrate la fonte di ispirazione di tutti gli scritti dialogici che invasero il campo editoriale dell'epoca come un nuovo genere letterario, «il faut en conclure que Socrate est le créateur du genre littéraire "dialogue socratique"» (p. 19); e di qui far scaturire l'indicazione di metodo che permette di identificare nel comportamento del Socrate personaggio, primo titolare e sempre protagonista dell'azione dialogica, i tratti caratterizzanti che tanto impressionarono i suoi contemporanei.

Ma la chiave di lettura più inquietante, che Roustang indica al lettore come effetto sorprendente e rivelatore della ricerca socratica di Rossetti, è l'irriverenza con cui lo studioso si rivolge al suo oggetto, sovvertendo il ritratto più paludato del mito platonico e scoprendo aspetti meno rassicuranti all'interno della retorica del dialogo. Si tratta della tesi che Rossetti enuncia indagando Le coté inauthentique du dialoguer platonicien (saggio 7), e soffermandosi poi in più occasioni sugli effetti della «macroretorica» dei dialoghi, ovvero sulle strutture architettoniche di scena dei testi, che creano un'atmosfera di plausibilità intorno al dialogare socratico, catturando gli interlocutori e il lettore in un gioco che appare non sempre onesto, dal punto di vista logico e educativo. L'effetto rivelatore, ma anche la produttività interpretativa di queste osservazioni di Rossetti, emergono con chiarezza attraverso il confronto tra i modi della rappresentazione platonica e quelli di Senofonte. Testo chiave nel contesto della raccolta, L'Euthydème de Xénophon (saggio 2) prende in esame un'unità dialogica del IV libro dei Memorabili (denominata da Rossetti Eutidemo per accostamento all'omonimo dialogo di Platone), leggendo in esso un comportamento socratico impossibile da catalogare come forma di paideia cognitiva, o anche soltanto gioco benevolo, con l'interlocutore: Eutidemo viene sopraffatto, prevaricato e distrutto da una marea di contro-esempi ad ogni tentativo, pur sensato, di fornire una risposta plausibile all'interrogazione di Socrate; di più, viene circuito entro una strategia complessa, che si sviluppa in diversi tempi e vede complici gli amici di Socrate nell'attirare al discorso il giovane studioso, che sembra non volerne sapere di riconoscere ad altri il ruolo di maestro, ma, una volta entrato nella trappola del dialogo, non può più sfuggire all'ansia e all'insicurezza generate in lui dall'insaziabile accanimento di Socrate. «Cruauté mentale ou acte suprême de confiance en Euthydème?», si domanda Rossetti (p. 89). In effetti, convinto a disprezzare se stesso e abbandonato senza una parola di conforto, Eutidemo troverà, secondo il racconto di Senofonte, la forza di reagire accettando il magistero socratico e sforzandosi di imitare il suo esempio; e sarà così (solo così) ben accolto nella cerchia dei socratici. Che cosa se ne può concludere? 
L'aspetto inquietante che si concentra in questo resoconto senofonteo non comporta in realtà un cambiamento di paradigma rispetto all'exemplum Socratis che la tradizione ha riconosciuto nel Socrate platonico: non è difficile rintracciare anche in Platone chiari segni di stile non benevolo nella paideia socratica, che inducono a sospettare di un'ermeneutica che «a si fréquemment fait passer Socrate pour un champion du dialogue paritaire» (p. 93). In realtà, sottolinea Rossetti, nella propensione del personaggio Socrate a instaurare rapporti non paritari, a mettere in difficoltà l'interlocutore spingendolo all'aporia, dovremmo semplicemente riconoscere «un fait d'observation» (p. 93), che accomuna le rappresentazioni dei socratici ${ }^{2}$. A esiti simili giunge infatti l'articolata ricognizione condotta nel saggio 6 (La rhétorique de Socrate), che sviluppa l'analisi in riferimento al Gorgia di Platone. Ciò che rende particolarmente interessante l'unità dialogica dell'Eutidemo di Senofonte (anche per comprendere meglio lo sfondo referenziale della rappresentazione di Platone) è che l'esito aporetico ha qui evidentemente l'aspetto di una lezione di metodo, escludendo ogni obiettivo di contenuto, ogni esito di carattere cognitivo. È dunque a una «lecture métacognitive du dialogue» che Rossetti guida il lettore, additando nel tipo di esperienza indotta dall' interrogare socratico il significato psicagogico della sua inusitata paideia.

Di quale esperienza si tratta? «L'expérience de la confusion, de l'embarras, de la honte», riassume Roustang, collegando questo metodo all'idea che la filosofia, per Socrate, sia «une pratique qui réclame une modification de la manière de vivre» (pp. 20-21). Sul significato più profondo e drammatico dell'esperienza provocata da Socrate nei suoi interlocutori, molto lavoro comparativo resta da fare. Il discorso potrebbe continuare a lungo esplorando la convergenza e la intertestualità delle scritture di Senofonte e Platone su questo specifico tratto di stile, che ciascun autore indirizza poi a esiti filosofici differenti.

Rossetti ne è in generale ben consapevole, mantenendo aperto lo spazio al confronto anche quando è impegnato a dimostrare l'originalità dell'elaborazione senofontea. È il caso del saggio 3, dedicato al capitolo III 8 dei Memorabili, dove Aristippo è ritratto nel tentativo di imitare il gioco confutatorio di Socrate, rubandogli il ruolo di «questionneur», mentre Socrate sembra impegnato a ripristinare a suo favore l'asimmetria del rapporto tra chi interroga e chi è interrogato. Dal punto di vista della rappresentazione, la scena appare singolare, e con pochi documenti comparabili, come «portrait du portrait» (p. 119): duplicazione del modello socratico sulla scena, attraverso la rappresentazione di un imitatore ancora maldestro. Ma la possibilità che si dia di Socrate vivente una imitazione tecnicamente riconoscibile è un fatto di per sé interessante per la storia del socratismo. Il riferimento agli imitatori, in particolare ai giovani, capaci di apprendere e riprodurre in tempi rapidi le mosse-chiave dell'elenchos, è ben presente anche in Platone e testimonia, come Rossetti sottolinea a proposito del testo di Senofonte, l'esistenza di regole precise «pour la joute dialectique à la Socrate» (p. 114).

\footnotetext{
2 Basterebbero, credo, gli esempi forniti nel del Lachete, e il commento in questo senso dal personaggio Nicia sulla strategia di Socrate nel dialogo (La. 187 e-188 a).
} 
Anche l'uso del ridicolo nelle mani di Socrate e dei suoi allievi, cui è dedicato il saggio 5 , si presta a considerazioni simili, in quanto elemento presente in modo trasversale nelle rappresentazioni dei socratici. Esaminandone e confrontandone diversi esempi, Rossetti ne mostra il potenziale agonale, che ne fa un sostegno inseparabile dall'elenchos, in quanto prova di valore, capace di mettere a rischio in modo permanente la credibilità di chi ne è vittima. Per quanto innovativo, e inserito in una cornice di rapporto assai più complessa di un'esibizione retorica, l'uso che Socrate ne fa resta crudele, in una cultura dove il riso è la più temuta fonte di vergogna e nessuno vorrebbe perdere un confronto faccia a faccia. L'induzione del ridicolo, l'ironia, la confutazione accanita, fanno di Socrate un provocatore di successo, ben consapevole del disagio prodotto nei suoi interlocutori sociali, come appare nell'Apologia platonica. Ma, se il socratismo è inseparabile dal "valore aggiunto" dei suoi effetti comici, è la forza delle sue istanze, «ce tissu d’idées de grande portée qui donne une force dévastatrice au ridicule socratique» (p. 213). Dal riso, siamo risospinti a indagare la serietà di alcune questioni emergenti dal contesto epocale delle rappresentazioni.

Ho lasciato per ultimo il commento al saggio 4, dedicato all'Eutifrone di Platone, perché mi permette di svolgere qualche considerazione conclusiva più personale sulla rilevanza del lavoro di Rossetti per chi è interessato al fenomeno della letteratura socratica come evento di portata filosofica, non riducibile alla ricerca di una dottrina.

Il titolo del saggio, L'Eutiphron comme événement communicationnel, ci indirizza in prima battuta a prendere sul serio l'intenzione comunicativa di Platone. A quali lettori mirava e quali effetti intendeva produrre in loro, considerato che il dialogo nasce in un ben preciso contesto storico e culturale, caratterizzato dal conflitto tra accusatori e difensori di Socrate dopo la sua morte e dalle dispute interne tra socratici per l'eredità autentica del maestro? L'ampia e sottile analisi, che Rossetti fa partire da questa domanda, scandaglia ogni aspetto della composizione dialogica, utilizzando diversi strumenti di esegesi e di comparazione intertestuale per mostrare, da un lato, ciò che accomuna questo testo ad altri dialoghi (permettendo di classificarlo tra quelli considerati “aporetici”), dall'altro ciò che lo rende in qualche modo unico e particolarmente inquietante, per la difficoltà a decifrare la strategia rappresentativa impiegata in esso da Platone.

Se l'argomento non fosse così scottante (la pietà, come virtù relativa al rapporto con gli déi), se l'interlocutore messo in imbarazzo non fosse un rappresentante scrupoloso della cultura religiosa cittadina, se l'occasione non fosse così drammatica (Socrate si sta recando al tribunale per conoscere le accuse formulate contro di lui), si potrebbe considerare il dialogo un esempio, tra gli altri, di uso del metodo confutatorio, applicato ai danni di un presunto competente in materia, da parte di un Socrate non particolarmente interessato all'argomento, ma solo a migliorare la tecnica della definizione (che Rossetti considera una peculiarità del Socrate platonico). Nella fattispecie, la pars destruens del lavoro di indagine, condotta con il consueto gusto del porre in imbarazzo mascherato da desiderio di apprendere, conduce Socrate non solo a mostrare che Eutifrone il sacerdote non ha un'idea precisa di ciò che identifica e caratterizza l'essere pii, ma a far intuire al lettore che è impossibile procedere oltre nel tentativo di definire la pietà (hosiotes) 
come virtù specifica, capace di spiegare e giustificare la benevolenza degli déi. È l'effetto di quel «morceau de bravoure», in cui Socrate dimostra che il pio non è pio perché è amato dagli dèi, ma per qualche altra ragione, mentre il prosieguo del dialogo dimostrerà che tale ragione non può essere legata alla relazione di servizio con gli dèi (che non hanno bisogno di doni), ma semmai con la giustizia. La conclusione è eminentemente negativa; ma la sparizione della pietà dal contesto delle virtù definibili in sé appare ben più che un esito aporetico. Come Rossetti sottolinea, «la question est bien plus cruciale que celle de la nature exacte du courage, de la modération ou de la beauté» (p. 181). E va a suo merito di "socratico" aver posto in dubbio «l'aporéticité effective ou seulement apparente» di questo dialogo, osservando che «Platon parvient à esquisser une réponse, même s'il la communique de façon si réticente et altérée qu'il la rende méconnaissable» (p. 187).

Ma come è possibile che Platone attribuisca al maestro di cui vuole salvare la memoria questa risposta così lacerante per i presupposti della cultura religiosa cittadina? Come è possibile che lo faccia, si domanda Rossetti, «surtout si l'on considère l'insertion explicite du dialogue dans la phase préliminaire du procés intenté à Socrate pour irréligion?» (p. 191). A risultare inquietante è per noi a questo punto soprattutto la strategia rappresentativa di Platone nei confronti di Socrate, che non rientra negli schemi difensivi praticati da altri e pare sostanzialmente inopportuna per un socratico.

Forse, per Platone contava rappresentare l'ultimo atto di un Socrate provocatore pubblico, votato al fraintendimento di fronte al ritualismo perbenista dei suoi concittadini. Forse questa frattura definitiva con le idee irriflesse di una cultura religiosa svuotata di forza morale era per Platone la premessa radicale (l'idea forte) che dava valore al fare filosofia alla maniera provocatoria di Socrate. Sicuramente il seguito ideale di questa espulsione della pietà dal novero delle virtù del cittadino sta nella fondazione laica del pensiero della giustizia, perseguita programmaticamente nella Repubblica come un bene che prescinde dal giudizio degli déi e dal desiderio di essere loro cari.

La cura attenta che Rossetti ha dedicato a questo dialogo ci restituisce l'Eutifrone come momento di svolta nella produzione platonica, che, dall'intenzione prevalentemente mimetica, destinata a trasmettere l'immagine e il metodo della ricerca di Socrate, passa a mettere a frutto autonomamente le più potenti tensioni innovative da lui suscitate. Il modo in cui lo studioso giunge a questi risultati, la ricchezza metodologica e l'apertura sempre in progress della sua analisi mi sembrano in qualche modo emblematiche della sua attiva e insostituibile presenza, nella stagione di studi di cui è stato ed è protagonista. 